\title{
In Vivo Studies of Phenylalanine Hydroxylase by Phenylalanine Breath Test: Diagnosis of Tetrahydrobiopterin-Responsive Phenylalanine Hydroxylase Deficiency
}

\author{
YOSHIYUKI OKANO, YUTAKA HASE, MIE KAWAJIRI, YASUAKI NISHI, KOJI INUI, \\ NORIO SAKAI, YOKO TANAKA, KAZUHIKO TAKATORI, MASAHIRO KAJIWARA, AND \\ TSUNEKAZU YAMANO \\ Department of Pediatrics [Y.O., M.K., Y.N., T.Y.], Osaka City University Graduate School of Medicine, \\ Osaka 545-8585, Japan; Nishinari Ward Public Health and Welfare Center [Y.H.], Osaka 557-8501, \\ Japan; Department of Developmental Medicine (Pediatrics) [K.I., N.S.], Osaka University Graduate \\ School of Medicine, Suita 565-0871, Japan; Department of Pediatrics [Y.T.], Tokyo Dental College \\ Ichikawa General Hospital, Ichikawa 272-8513, Japan; and Department of Medicinal Chemistry [K.T., \\ M.K.], Meiji Pharmaceutical University, Kiyose 204-8588, Japan
}

\begin{abstract}
Tetrahydrobiopterin $\left(\mathrm{BH}_{4}\right)$-responsive phenylalanine hydroxylase $(\mathrm{PAH})$ deficiency is characterized by reduction of blood phenylalanine level after a $\mathrm{BH}_{4}$-loading test. Most cases of $\mathrm{BH}_{4}$-responsive $\mathrm{PAH}$ deficiency include mild phenylketonuria (PKU) or mild hyperphenylalaninemia (HPA), but not all patients with mild $\mathrm{PKU}$ respond to $\mathrm{BH}_{4}$. We performed the phenylalanine breath test as reliable method to determine the $\mathrm{BH}_{4}$ responsiveness. Phenylalanine breath test quantitatively measures the conversion of $\mathrm{L}-\left[1-{ }^{13} \mathrm{C}\right]$ phenylalanine to ${ }^{13} \mathrm{CO}_{2}$ and is a noninvasive and rapid test. Twenty Japanese patients with HPA were examined with a dose of $10 \mathrm{mg} / \mathrm{kg}$ of ${ }^{13} \mathrm{C}$-phenylalanine with or without a dose of $10 \mathrm{mg} \cdot \mathrm{kg}^{-1} \cdot \mathrm{d}^{-1}$ of $\mathrm{BH}_{4}$ for $3 \mathrm{~d}$. The phenylalanine breath test [cumulative recovery rate (CRR)] could distinguish control subjects $(15.4 \pm 1.5 \%)$; heterozygotes $(10.3 \pm 1.0 \%)$; and mild HPA (2.74\%), mild PKU (1.13 \pm $0.14 \%)$, and classical PKU patients $(0.29 \pm 0.14 \%)$. The genotypes in mild PKU cases were compound heterozygotes with mild (L52S, R241C, R408Q) and severe mutations, whereas a
\end{abstract}

\section{ABSTRACT}

mild HPA case was homozygote of R241C. CRR correlated inversely with pretreatment phenylalanine levels, indicating the gene dosage effects on PKU. $\mathrm{BH}_{4}$ loading increased CRR from $1.13 \pm 0.14$ to $2.95 \pm 1.14 \%$ (2.6-fold) in mild PKU and from 2.74 to $7.22 \%$ (2.6-fold) in mild HPA. A CRR of 5 to $6 \%$ reflected maintenance of appropriate serum phenylalanine level. The phenylalanine breath test is useful for the diagnosis of $\mathrm{BH}_{4}$-responsive $\mathrm{PAH}$ deficiency and determination of the optimal dosage of $\mathrm{BH}_{4}$ without increasing blood phenylalanine level. (Pediatr Res 56: 714-719, 2004)

$\mathbf{B H}_{4}$, tetrahydrobiopterin,

CRR, cumulative recovery rate,

HPA, hyperphenylalaninemia,

PAH, phenylalanine hydroxylase,

PKU, phenylketonuria,

PTPS, 6-pyruvoyl-tetrahydropterin synthase
Phenylketonuria (PKU) is an autosomal recessive disorder caused by deficiency of hepatic phenylalanine hydroxylase (PAH; EC 1.14.16.1). The disease causes mental retardation unless the affected child is maintained on a strict low-

Received November 17, 2003; accepted May 24, 2004.

Correspondence: Yoshiyuki Okano, M.D., Department of Pediatrics, Osaka City University Graduate School of Medicine, 1-4-3 Asahimachi, Abeno-ku, Osaka 545-8585, Japan; e-mail: okano@med.osaka-cu.ac.jp

This study was supported in part by grants from the Ministry of Education, Culture Sports, Science, and Technology of Japan, and by fund from Suyama Research Foundation.

DOI: 10.1203/01.PDR.0000141520.06524.51 phenylalanine diet (1). Newborn mass screening for PKU is performed worldwide, and patients with a wide spectrum of clinical severity have been identified with almost $100 \%$ probability. The incidence of PKU in Japan is 1/120,000 (2) and is much lower than in whites $(1 / 10,000)$ (3) and Chinese (1/ 18,000) (4). PKU is a heterogeneous metabolic disorder at both clinical and genetic levels.

The diagnosis of PKU is based on the presence of high concentration of phenylalanine in the serum and lack of deficiency of tetrahydrobiopterin $\left(\mathrm{BH}_{4}\right)$, rather than by measuring hepatic PAH activity. The clinical severity of PAH deficiency is also determined mainly by serum phenylalanine level, al- 
though blood phenylalanine concentration is influenced by the dietary protein intake. For an appropriate diet therapy influenced by the social environments and personal situations, it is important to determine directly the clinical severity of PKU and PAH activity. As a representative autosomal recessive inherited disease, it is important to investigate how both alleles of the PKU gene influence PAH activity and how they give rise to clinical symptoms. We reported in 1991 that the clinical phenotype of PKU patients correlated with the genotype, which was the average of in vitro PAH activity of both mutations (5). However, subsequent studies reported that the clinical phenotype in several cases of PKU did not correspond with the genotype $(6,7)$. The metabolism of phenylalanine in the human body not only reflects PAH protein but also is influenced by many factors, such as absorption and excretion of phenylalanine and the regulation of transcription and/or translation on PAH gene.

Various methods are available for in vivo measurement of PAH activity. ${ }^{2} \mathrm{H}$-tyrosine in blood is measured after administration of ${ }^{2} \mathrm{H}$-phenylalanine using gas chromatography mass spectrometry. The results of analysis using this method correlated with hepatic PAH activity and clinical phenotype (8-10). However, this method requires the use of a large amount of phenylalanine as a loading dose $(10-200 \mathrm{mg} / \mathrm{kg})$ and frequent blood sampling. Treacy et al. (11) described the phenylalanine breath test, a rapid noninvasive test for measurement of the actual phenylalanine tolerance. The test is based on quantitative measurement of the conversion of $\mathrm{L}-\left[1-{ }^{13} \mathrm{C}\right]$ phenylalanine to ${ }^{13} \mathrm{CO}_{2}$ through tyrosine by $\mathrm{PAH}$. The phenylalanine breath test provides information on the whole-body phenylalanine oxidative capacity, as an index of in vivo PAH activity.

Recently, $\mathrm{BH}_{4}$-responsive PAH deficiency was characterized by a decrease of blood phenylalanine after a $\mathrm{BH}_{4}$ loading test (12) and patients with this deficiency have been treated with long-term $\mathrm{BH}_{4}$ (13-17). Most patients of $\mathrm{BH}_{4}$-responsive PAH deficiency have mild PKU and mild hyperphenylalaninemia (HPA), but not all patients with mild PKU respond to $\mathrm{BH}_{4}$. In fact, even patients with similar mild mutations exhibit different response to $\mathrm{BH}_{4}(12,17)$. Using the phenylalanine breath test, Muntau et al. (17) reported recently that $\mathrm{BH}_{4}$ increases $\mathrm{PAH}$ activity in patients with $\mathrm{BH}_{4}$-responsive $\mathrm{PAH}$ deficiency. The present study was designed to determine whether phenylalanine oxidation capacity is consistent with the clinical phenotype and genotype and whether determination of this parameter is useful for the diagnosis of $\mathrm{BH}_{4}$-responsive PAH deficiency.

\section{METHODS}

Subjects. The subjects of this study were 20 Japanese patients (11 male individuals, aged 1-23 y) who were confirmed to have PAH deficiency on the basis of clinical and biochemical evaluation at the participating institutions. The patients, except for an adult patient with mild and another with classical $\mathrm{PKU}$, were on phenylalanine-free milk and low-protein food ranging from insufficient to sufficient. Serum phenylalanine levels measured before the breath test in the two untreated patients with mild and classical PKU were 0.97 and $1.45 \mathrm{mM}$, respectively. The mean serum phenylalanine concentrations were $0.31 \pm 0.14 \mathrm{mM}( \pm \mathrm{SD}$; range: $0.11-0.46 \mathrm{mM})$ in six treated patients with mild PKU and HPA and $0.43 \pm 0.28 \mathrm{mM}$ (range: $0.068-0.95 \mathrm{mM}$ ) in 12 treated patients with classical PKU. Two patients with 6-pyruvoyl-tetrahydropterin synthase (PTPS) deficiency were diagnosed by analysis of urinary pteridine, biopterin loading test, and measurement of PTPS activity and treated with $\mathrm{BH}_{4}, 5$-hydroxytryptophan, L-dopa, and carbidopa. The criterion for classical PKU is serum phenylalanine concentration of $\geq 1.2 \mathrm{mM}$ before initiation of a phenylalaninerestricted diet or in the absence of dietary restrictions later in life. The serum phenylalanine concentrations in mild PKU and mild HPA were $0.6-1.2 \mathrm{mM}$ and $<0.6 \mathrm{mM}$, respectively, without phenylalanine-restricted diet. Serum phenylalanine was measured using ion-exchange chromatography.

Phenylalanine breath test. For phenylalanine breath test, $99 \%$ enriched $\mathrm{L}-\left[1-{ }^{13} \mathrm{C}\right]$ phenylalanine was administered orally at a dose of $10 \mathrm{mg} / \mathrm{kg}$ and a maximum of $200 \mathrm{mg}$ after overnight fast. Breath samples were collected into aluminum bags at $0,10,20,30,45,60,90$, and 120 min. $6 \mathrm{R}^{-\mathrm{BH}_{4}}$ (Suntory Co., Tokyo, Japan) was administered orally at a dose of 10 $\mathrm{mg} / \mathrm{kg}$ with a maximum of $200 \mathrm{mg}$, divided into two doses per day at -2 and $-1 \mathrm{~d}$ and another dose of $10 \mathrm{mg} / \mathrm{kg}$, with a maximum of $200 \mathrm{mg}, 3 \mathrm{~h}$ before the breath test, as shown in Figure 1. The breath test was repeated twice in seven patients (two classical PKU and five mild PKU), before and after $\mathrm{BH}_{4}$. The administered ${ }^{13} \mathrm{C}$-phenylalanine is metabolized by PAH and is exhaled as ${ }^{13} \mathrm{CO}_{2} \cdot{ }^{12} \mathrm{CO}_{2}(\mathrm{~m} / \mathrm{z} 44)$ and ${ }^{13} \mathrm{CO}_{2}(\mathrm{~m} / \mathrm{z} 45)$ were measured using gas chromatograph/mass spectrometer (Breath MAT Plus; Finnigan MAT, Bremen, Germany) (18). Results of the ${ }^{13} \mathrm{CO}_{2}$ breath tests were expressed as ${ }^{13} \mathrm{CO}_{2}$ excess permillage $\left(\Delta^{13} \mathrm{C}, \%\right.$ o $)$ and cumulative recovery rate (CRR; \%) (11). The $\Delta^{13} \mathrm{C}(\%)$ was expressed as the ratio of ${ }^{13} \mathrm{CO}_{2} /{ }^{12} \mathrm{CO}_{2}$. The CRR was expressed as the ratio of total amounts of ${ }^{13} \mathrm{CO}_{2}$ (moles) in expiration during $120 \mathrm{~min}$ for administered dose of ${ }^{13} \mathrm{C}$-phenylalanine. Total $\mathrm{CO}_{2}$ production speed is calculated from the body surface area $\left(5 \mathrm{mmol} \cdot \mathrm{m}^{-2}\right.$ - $\left.\min ^{-1}\right)(19)$, and the body surface area (BSA; in $\mathrm{m}^{2}$ ) is calculated by the formula [body weight $(\mathrm{kg})^{0.5378} \times$ height $\left.(\mathrm{cm})^{0.3964} \times 0.024265\right](20)$. Serum phenylalanine was measured before administration of $\mathrm{L}-\left[1-{ }^{13} \mathrm{C}\right]$ phenylalanine and $1 \mathrm{~h}$ after administration using Hitachi automatic amino acid analyzer L8800 (Hitachi Co., Hitachinaka, Japan).

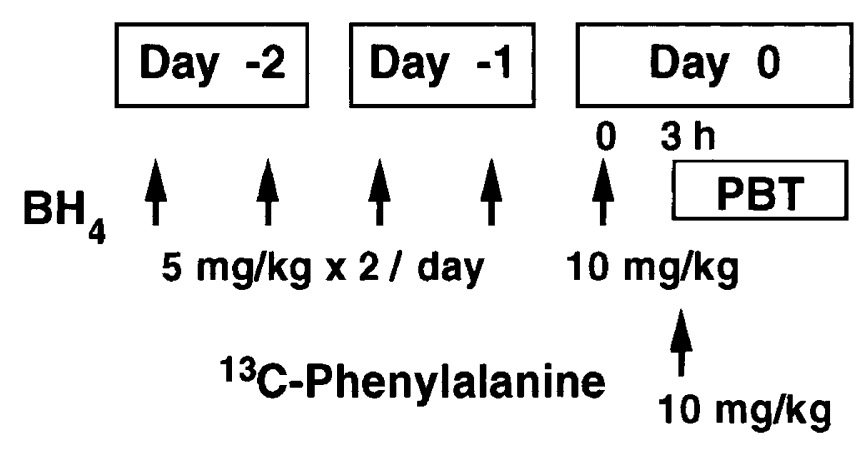

Figure 1. Time schedule of ${ }^{13} \mathrm{C}$-phenylalanine ingestion and $\mathrm{BH}_{4}$ dosing. PBT, phenylalanine breath test. 
DNA analysis. PAH mutations were determined by using denaturing gradient gel electrophoresis and DNA sequencing, as described previously $(21,22)$. Genomic DNA was isolated from lymphocytes or EBV transformed lymphoblasts. Thirteen exons and flanking intronic regions of the $\mathrm{PAH}$ gene were amplified by PCR with GC-clamped primers according to Guldberg et al. (23). The target exons with mutations were amplified from genomic DNA by PCR with biotinylated primers and were purified to single-strand DNA using magnetic beads coated with streptavidin M280 (Dynal, Oslo, Norway). The purified single-stranded DNA was sequenced by the dye terminator method using an ABI PRISM 310 Genetic Analyzer (Perkin Elmer, Norwalk, CT).

Statistical analysis and ethical issues. All data were expressed as mean \pm SD unless otherwise stated. Differences between groups were examined for statistical significance using the $t$ test. A $p<0.05$ denoted the presence of a statistically significant difference. Statistical analyses were performed using the Statview program version 4.5 (Abacus Concepts, Berkeley, CA).

All protocols described in the above studies were approved by the institutional review boards of Osaka City University Graduate School of Medicine, and informed consent for the breath test and genetic analysis was obtained from all patients or their parents.

\section{RESULTS}

Identification of genotype. Table 1 shows the results of genetic analysis of 13 patients with classical PKU, six patients with mild PKU, and one patient with mild HPA. A total of 18 mutations were identified in 40 PKU alleles of the 20 patients, except for one allele. Four mutations of A202V (GCT $\rightarrow$ GTT), R252P (CGG $\rightarrow$ CCG), Q301H (CAG $\rightarrow$ CAT), and $\mathrm{D} 415 \mathrm{H}$ (GAC $\rightarrow$ CAC) have not been reported. The genotypes

Table 1. Genotypes and in vitro PAH activity in 20 patients with PAH deficiency

\begin{tabular}{lccc}
\hline Genotype & $n$ & $\%$ & PAH activity (\%) \\
\hline Severe type & & & \\
R413P & 12 & 30 & 0 \\
IVS4nt-1 & 3 & 7.5 & 0 \\
R111X & 2 & 5 & 0 \\
De15\&6 & 2 & 5 & $1^{(22)}$ \\
T278I & 2 & 5 & 0 \\
Y77X & 1 & 2.5 & ND \\
A202V & 1 & 2.5 & 0 \\
E6nt-96a $>$ g & 1 & 2.5 & $10^{(30)}$ \\
R243Q & 1 & 2.5 & ND \\
R252P & 1 & 2.5 & 0 \\
R261X & 1 & 2.5 & 0 \\
IVS10nt-14 & 1 & 2.5 & ND \\
Q301H & 1 & 2.5 & ND \\
D415H & 1 & 2.5 & \\
L430P & 1 & 2.5 & $25^{(25)}$ \\
Mild type & & & $27^{(19)}$ \\
R241C & 6 & 15 & $55^{(26)}$ \\
L52S & 1 & 2.5 & \\
R408Q & 1 & 2.5 & \\
Total & $39 / 40$ & 97.5 &
\end{tabular}

$\mathrm{ND}$, not determined. in mild PKU cases were from compound heterozygotes with mild (L52S, R241C, R408Q) and severe mutations, whereas in the mild HPA case, it was homozygote of mild mutation (R241C/R241C). In this study, we could not find cases with discordance between genotype and clinical phenotype.

Serum phenylalanine concentration. We also examined the influence of ${ }^{13} \mathrm{C}$-phenylalanine loading $(10 \mathrm{mg} / \mathrm{kg}$; maximum $200 \mathrm{mg}$ ) on serum phenylalanine concentration. No large increase was noted in serum phenylalanine concentration before and after administration of ${ }^{13} \mathrm{C}$-phenylalanine in both classical PKU and mild PKU/HPA patients (an increase from a predosing value of $0.51 \pm 0.39$ to $0.62 \pm 0.38 \mathrm{mM}$ at $1 \mathrm{~h}$ after phenylalanine dosing and from $0.41 \pm 0.29$ to $0.44 \pm$ $0.26 \mathrm{mM}$, respectively).

${ }^{13} \mathrm{CO}_{2} /{ }^{12} \mathrm{CO}_{2}$ ratio in breath test. Figure 2 shows the changes in ${ }^{13} \mathrm{CO}_{2} /{ }^{12} \mathrm{CO}_{2}$ ratio $\left(\Delta^{13} \mathrm{C}\right)$ in expired air. The peak level of $\Delta^{13} \mathrm{C}$ in the control occurring between 20 and $30 \mathrm{~min}$ after dosing (42.3 \pm 10.4 ; range: $28.7-53.4 \%$ ) was significantly higher than that of the heterozygotes at the same time interval $(27.2 \pm 6.0 ; 21.1-32.5 \%$; $p<0.05)$. These results indicated that the administered ${ }^{13} \mathrm{C}$-phenylalanine dose reached the liver at 20-30 min after dosing, where it was mainly metabolized to tyrosine, $p$-hydroxyphenylpyruvate, and homogentisic acid in the liver with a first-pass effect. Patients with mild HPA showed a small peak of $\Delta^{13} \mathrm{C}$ at $45 \mathrm{~min}$, reflecting the residual PAH activity. Low PAH activity was observed in classical PKU and mild PKU, and no peaks were noted for $\Delta^{13} \mathrm{C}$. After $\mathrm{BH}_{4}$ loading, mild PKU showed a peak $\Delta^{13} \mathrm{C}$ level of $8.87 \pm 8.99 \%$ (range: $3.83-21.62 \%$ ) at $20-30$ min. Mild HPA showed a peak $\Delta^{13} \mathrm{C}(24.45 \%$ ) at $20-30 \mathrm{~min}$, a pattern similar to that noted in heterozygotes. $\mathrm{BH}_{4}$ loading markedly improved phenylalanine oxidation in mild PKU and mild HPA.

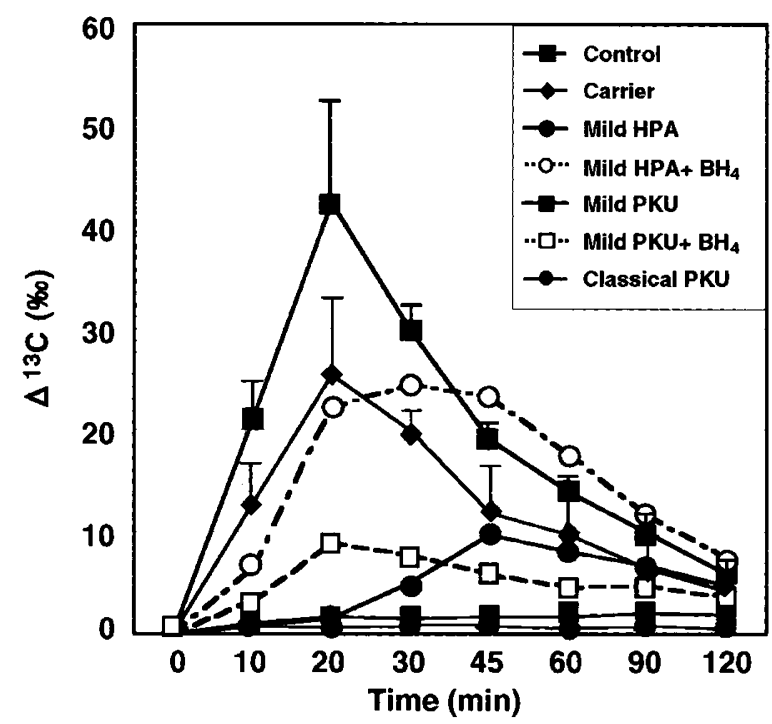

Figure 2. Time course of ${ }^{13} \mathrm{CO}_{2}$ excretion during phenylalanine breath test in control subjects, heterozygotes, and patients with PAH deficiency. $\Delta^{13} \mathrm{C}(\% \circ)$ values during $120 \mathrm{~min}$ after ingestion of ${ }^{13} \mathrm{C}$-phenylalanine are expressed as solid lines without $\mathrm{BH}_{4}$ dosing and as dashed lines with $\mathrm{BH}_{4}$ dosing. Data of the control and heterozygotes are expressed as mean $\pm \mathrm{SD}$, whereas those of patients with PAH deficiency are expressed as mean values. 
CRR in breath test. As shown in Figure 3, the phenylalanine breath test exhibited continuous levels of CRR in PAH deficiency from 0 (classical PKU) to $2.74 \%$ (mild HPA). These results are in agreement with various clinical manifestations of PKU. The CRR (in vivo PAH activity) could distinguish control subjects (15.4 $\pm 1.5 \%$; range: $13.79-17.44 \%)$; the heterozygotes (10.3 $\pm 1.0 \%$; $9.00-11.42 \%)$; and mild HPA $(2.74 \%)$, mild PKU $(1.13 \pm 0.14 \% ; 1.01-1.40 \%)$, and classical PKU (0.29 $\pm 0.14 \% ; 0-0.93 \%)$ patients (Fig. 3). We found consistency between the CRR and clinical phenotype in the Japanese patients who were tested in this study. Patients with compound heterozygotes of mild (L52S, R241C, R408Q) and severe mutations had CRR of $1.0-1.4 \%$ and mild PKU phenotype, whereas patients with severe mutations for both alleles had CRR of $<0.93 \%$ and classical PKU phenotype. In this study, we did not find inconsistency among clinical phenotype, CRR, and genotype.

$\mathrm{BH}_{4}$ loading increased CRR from $1.13 \pm 0.14$ to $2.95 \pm$ $1.14 \%$ (2.6-fold) in all four patients with mild PKU and also increased it from 2.74 to $7.22 \%$ (2.6-fold) in mild HPA patients. All patients with mild PKU and mild HPA in this study responded to $\mathrm{BH}_{4}$. Two patients with classical PKU showed no increase in the $\mathrm{CRR}$ after $\mathrm{BH}_{4}$ loading. $\mathrm{BH}_{4}$-induced activation was proportional to residual PAH activity. In PTPS patients 1 and 2 , serum phenylalanine was effectively controlled to $\leq 0.12$ $\mathrm{mM}$ after administration of $\mathrm{BH}_{4}$ at 3.4 and $6 \mathrm{mg} \cdot \mathrm{kg}^{-1} \cdot \mathrm{d}^{-1}$, respectively, with regular food. The CRR values in these two patients were 5.88 and $19.0 \%$, respectively.
Correlation between CRR and phenylalanine levels without dietary treatment. Correlation between CRR and serum phenylalanine levels without dietary treatment was examined in 26 patients. Serum phenylalanine levels of four control subjects, four heterozygotes, and two patients with PTPS deficiency were measured before administration of phenylalanine in the breath test. Plasma phenylalanine levels of 16 patients with PAH deficiency were examined before phenylalaninerestriction therapy. As shown in Figure 4, CRR correlated inversely with phenylalanine concentration $(1 / \mathrm{y}=0.69+$ $1.02 \mathrm{x} ; p<0.0001)$. This result indicates that phenylalanine levels can decrease steeply with a slight increase of CRR (from $0 \%$ or near $0 \%$ to $1-2 \%$ ), and the clinical phenotype changes from classical PKU to mild HPA.

\section{DISCUSSION}

In the phenylalanine breath test, the administered ${ }^{13} \mathrm{C}$ phenylalanine is absorbed in the intestine and transported to the liver cells through the portal vein. In the liver, ${ }^{13} \mathrm{C}$ phenylalanine is converted to ${ }^{13} \mathrm{C}$-tyrosine by $\mathrm{PAH}$, then to homogentisic acid by tyrosine aminotransferase and dioxygenase, and is finally exhaled as ${ }^{13} \mathrm{CO}_{2}$. Thus, this test not only simply measures PAH activity but also evaluates the overall state of phenylalanine metabolism in humans, i.e. phenylalanine oxidation capacity. This test is expected to reflect the clinical phenotype of PKU. The phenylalanine breath test used in the present study does not require blood sampling or special

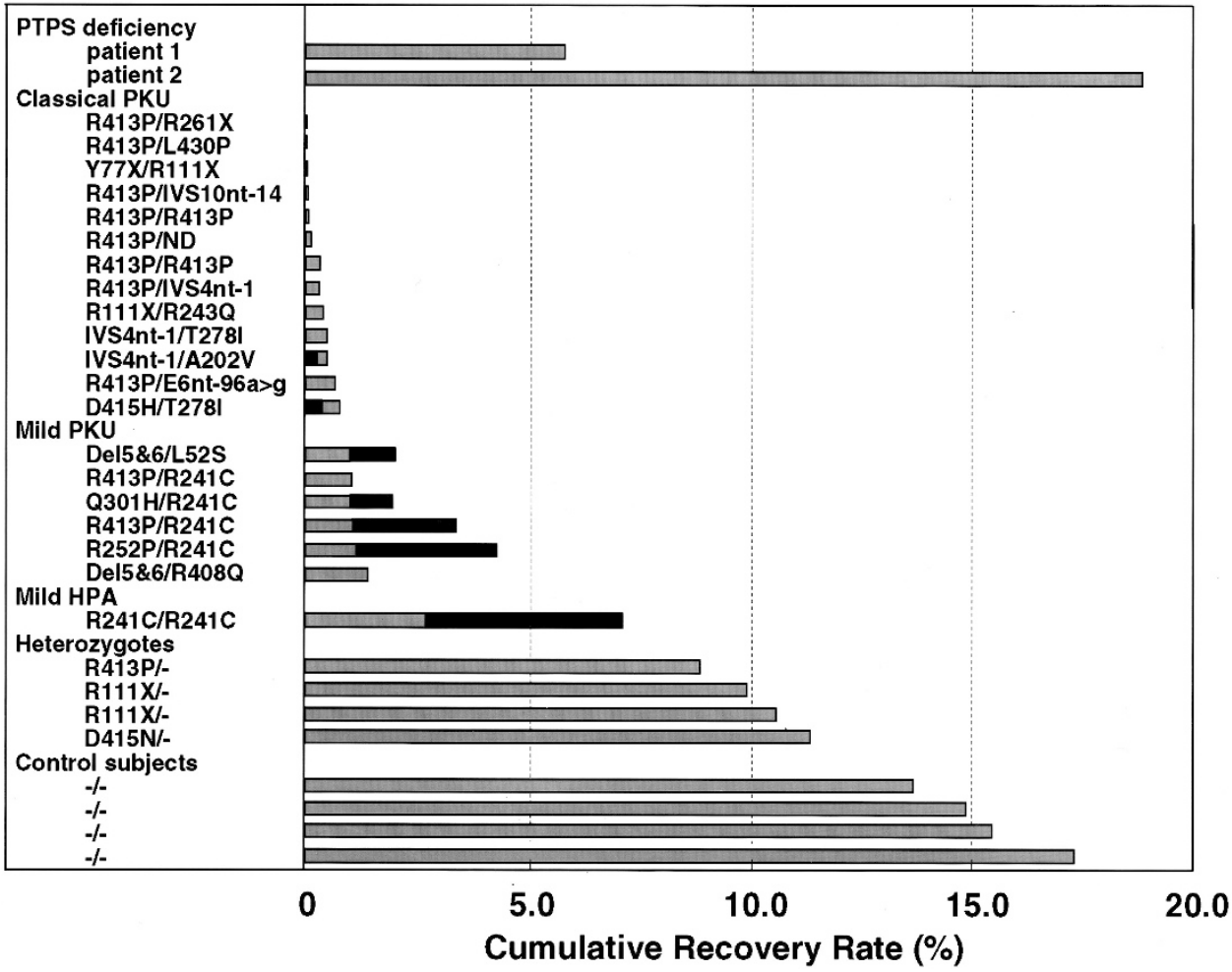

Figure 3. Phenylalanine oxidation capacity in control subjects, heterozygotes, patients with PAH deficiency, and patients with PTPS deficiency. 盽, CRR (\%) values determined during $120 \mathrm{~min}$ after the ingestion of ${ }^{13} \mathrm{C}$-phenylalanine without $\mathrm{BH}_{4}$ dosing; $\boldsymbol{\square}$, CRR (\%) values determined during 120 min after the ingestion of ${ }^{13} \mathrm{C}$-phenylalanine with $\mathrm{BH}_{4}$ dosing. The detected mutations in $\mathrm{PAH}$-deficient patients and heterozygotes are indicated in the left panel. -, no mutation; ND, not determined. 


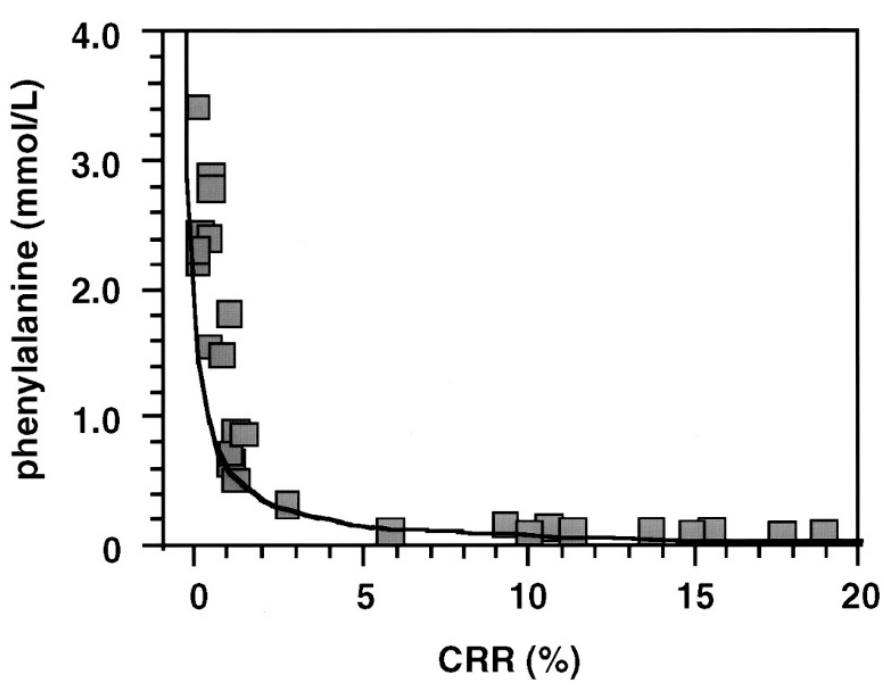

Figure 4. Correlation between pretreatment phenylalanine levels and CRR in control subjects, heterozygotes, patients with PAH deficiency, and patients with PTPS deficiency $(n=26)$.

conditions such as high serum phenylalanine levels. The results are deduced from analysis of the expired gas collected over a short period of time. Thus, the phenylalanine breath test is simple and noninvasive and can be repeated several times.

The CRR in phenylalanine breath test showed a continuum from classical PKU to mild HPA (Fig. 3). This finding is in agreement with the clinical notion that PKU is a highly heterogeneous disease for the clinical phenotype, which is caused by the strong heterogeneity of PKU mutations. In fact, 18 different mutations were detected in 20 patients in the present study. Patients with classical PKU, mild PKU, and mild HPA had significantly different CRR values of $<1 \%, 1-1.4 \%, 2.4 \%$, respectively. Therefore, we can predict the clinical phenotype from the CRR. Furthermore, CRR can be used to determine adherence to diet therapy. Concerning the effects of $\mathrm{BH}_{4}$ on PAH, all five patients with mild PKU and mild HPA responded to $\mathrm{BH}_{4}$ loading and showed an increase in CRR, which was proportional to the residual $\mathrm{PAH}$ activity after $\mathrm{BH}_{4}$ dosing. In patients with classical $\mathrm{PKU}, \mathrm{BH}_{4}$ loading did not increase CRR. When the CRR is $1 \%$ or higher before $\mathrm{BH}_{4}$ dosing, the response to $\mathrm{BH}_{4}$ may be expected (Fig. 3). A CRR value of $5.88 \%$ in patients with PTPS deficiency indicated $<0.12 \mathrm{mM}$ of serum phenylalanine levels on long-term $\mathrm{BH}_{4}$ therapy. These findings suggest that the cumulative recovery of 5 to $6 \%$ is also sufficient for maintaining serum phenylalanine level $(0.12 \mathrm{mM})$ in patients with PAH deficiency and that our test may be potentially useful for determining the optimal dosage of $\mathrm{BH}_{4}$ for long-term medication of $\mathrm{BH}_{4}$-responsive HPA patients.

The frequency and types of PKU mutations differ greatly between whites and East Asians $(21,24)$. Mutations associated with mild phenotype and $\mathrm{BH}_{4}$-responsive $\mathrm{PAH}$ deficiency in East Asians also differ from those in whites. In the present study, all patients with mild PKU phenotype were compound heterozygotes with severe and mild mutations, which included R241C (in vitro PAH activity; 25\%) (25), L52S (27\%) (22), and R408Q (55\%) (26), as shown in Table 1. Mild HPA was
R241C homozygote. The patient with R408Q (in vitro PAH activity, 55\%) and Del 5\&6 had higher CRR than four patients with R241C (in vitro PAH activity, 25\%) and severe mutations (R413P, Q301H, and R252P). The phenylalanine oxidation capacity, i.e. CRR, determined by our test stands between the clinical phenotype and the genotype and links them together. In the present study of Japanese patients, none of the patients showed any disagreement among clinical phenotype, phenylalanine oxidation capacity, and genotype. It follows that the genotype determined by both alleles mainly specifies $\mathrm{PAH}$ activity, which in turn specifies the clinical manifestations in an individual.

However, in East Asians, discordance between $\mathrm{BH}_{4}$ responsiveness and genotype has been reported (12). Two patients with P407S mutation were described, one as a nonresponder (P407S/R111X) and the other as a responder (P407S/R252W) to $\mathrm{BH}_{4}$. This different responsiveness is thought to be due to another mutation in each patient (R111X: stop codon mutation; R252W: missense mutation) on PAH protein synthesis. In whites, inconsistencies associated with $\mathrm{BH}_{4}$ responsiveness are reported concerning Y414C, L48S, I65V, and R261Q mutations, and the $\mathrm{BH}_{4}$ responders and nonresponders are present in individuals with the same mild mutation (17).

The cause for discordance among clinical phenotype including $\mathrm{BH}_{4}$-responsive $\mathrm{PAH}$ deficiency and genotype is not yet clear. In addition, the mechanism responsible for the recovery of defective $\mathrm{PAH}$ activity after $\mathrm{BH}_{4}$ loading remains elusive. Two broad factors determine the effect of $\mathrm{BH}_{4}$ on $\left.\mathrm{PAH}: 1\right) \mathrm{BH}_{4}$ site: absorption, distribution, and metabolism of orally administered $\mathrm{BH}_{4}$, and 2) $\mathrm{PAH}$ site: interaction between $\mathrm{BH}_{4}$ and $\mathrm{PAH}$ gene and protein. Concerning $\mathrm{BH}_{4}$ site, absorption of $\mathrm{BH}_{4}$ is minimal and unstable and differs greatly from one individual to another (Suntory Co., personal communication). The optimal dose and the duration of $\mathrm{BH}_{4}$ administration for the diagnosis of $\mathrm{BH}_{4}$-responsive $\mathrm{PAH}$ deficiency remain unknown. Bernegger et al. (27) pointed out that a single dose of $20 \mathrm{mg} / \mathrm{kg}$ of $6 \mathrm{R}^{-} \mathrm{BH}_{4}$, the active form, was 5-20 times more effective than smaller doses of $6 \mathrm{R}-\mathrm{BH}_{4}$ or $6 \mathrm{R}, \mathrm{S}_{-} \mathrm{BH}_{4}$ and induced a response in $70 \%$ of patients with mild $\mathrm{PAH}$ deficiency. With regard to the optimum $\mathrm{BH}_{4}$ dose for long-term control of patients with $\mathrm{BH}_{4}$-responsive $\mathrm{PAH}$ deficiency, favorable blood phenylalanine levels were obtained at a $\mathrm{BH}_{4}$ dose of $5-10 \mathrm{mg} / \mathrm{kg}$. It may be necessary to repeat $\mathrm{BH}_{4}$ doses over several days for unstable absorption of $\mathrm{BH}_{4}$. In our study using $\mathrm{BH}_{4}$ at $10 \mathrm{mg} \cdot \mathrm{kg}^{-1} \cdot \mathrm{d}^{-1}$ for $3 \mathrm{~d}$, a rise of PAH activity was noted in all patients with mild PKU and mild HPA.

Concerning PAH site factors, various mutations associated with $\mathrm{BH}_{4}$ responsiveness have been identified, and some mutations were outside the catalytic domain or the locusassociated $\mathrm{Km}$ variant for $\mathrm{BH}_{4}$ of the $\mathrm{PAH}$ enzyme. Direct effects of $\mathrm{BH}_{4}$ are suspected. In other words, $\mathrm{BH}_{4}$ may upregulate the expression of the $\mathrm{PAH}$ gene, stabilize $\mathrm{PAH}$ mRNA, and facilitate and stabilize the formation of functional PAH tetramers (16). Figure 4 provided in this study seems to confirm the proposal put forward by Scriver (28): "gene dosage effect in PAH deficiency." Figure 4 may clarify the causes and the mechanisms of the $\mathrm{BH}_{4}$ responsiveness in mild $\mathrm{PAH}$ deficiency and the discordance between genotype and clinical 
phenotype. Mutations that were identified in cases with discordance between genotype and clinical phenotype were basically related to the mild genotype. Patients with the mild clinical phenotype exist at the turning point of the correlation curve shown in Figure 4. This mild phenotype is produced by a small residual PAH activity, which is specified to the genotype. The formula suggests that a slight increase of the CRR at the turning point by certain effectors should greatly reduce blood phenylalanine level and cause transformation to a mild phenotype. In contrast, a slight decrease of the CRR leads to a rise in blood phenylalanine level and subsequently leads to transformation to a severe phenotype. Patients with mild mutation could become milder or more severe by certain effectors of the PAH enzyme. $\mathrm{BH}_{4}$ is advocated as a strong effector to influence mutations at the turning point.

The phenylalanine breath test is useful for the diagnosis of $\mathrm{BH}_{4}$-responsive $\mathrm{PAH}$ deficiency and determination of the optimal dosage of $\mathrm{BH}_{4}$ without increasing blood phenylalanine level. To clarify the discordance between clinical phenotype including $\mathrm{BH}_{4}$ responsiveness and genotype, it is important to investigate both genotype and phenylalanine oxidation capacity and to further accumulate such data.

\section{REFERENCES}

1. Scriver CR, Kaufman S 2001 Hyperphenylalaninemia: phenylalanine hydroxylase deficiency. In: Scriver CR, Baudet AL, Valle D, Sly WS (eds) The Metabolic and Molecular Bases of Inherited Disease, Vol II, 8th Ed. McGraw-Hill, New York, pp $1667-1724$

2. Aoki K, Wada Y 1988 Outcome of the patients detected by newborn screening in Japan. Acta Paediatr Jpn 30:429-434

3. Bickel H, Bachmann C, Beckers R, Brandt NJ, Clayton BE, Corrado G, Feingold HJ, Giardini O, Hammersen G, Schönberg D 1981 Neonatal mass screening for metabolic disorders. Eur J Pediatr 137:133-139

4. Liu SR, Zuo QH 1986 Newborn screening for phenylketonuria in eleven districts. Chin Med J (Engl) 99:113-118

5. Okano Y, Eisensmith RC, Güttler F, Lichter-Konecki U, Konecki DS, Trefz FK, Dasovich M, Wang T, Henriksen K, Lou H, Woo SL 1991 Molecular basis of phenotypic heterogeneity in phenylketonuria. N Engl J Med 324:1232-1238

6. Guldberg P, Rey F, Zschocke J, Romano V, Francois B, Michiels L, Ullrich K, Hoffmann G, Burgard P, Schmidt H, Meli C, Riva E, Dianzani I, Ponzone A, Rey J, Güttler F 1998 A European multicenter study of phenylalanine hydroxylase deficiency: classification of 105 mutations and a general system for genotype-based prediction of metabolic phenotype. Am J Hum Genet 63:71-79

7. Kayaalp E, Treacy E, Waters PJ, Byck S, Nowacki P, Scriver CR 1997 Human phenylalanine hydroxylase mutations and hyperphenylalaninemia phenotypes: a metanalysis of genotype-phenotype correlations. Am J Hum Genet 61:1309-1317

8. Matalon R, Matthews DE, Michals K, Bier D 1982 The use of deuterated phenylalanine for the in vivo assay of phenylalanine hydroxylase activity in children. J Inherit Metab Dis 5:17-19

9. Trefz FK, Erlenmaier T, Hunneman DH, Bartholome K, Lutz P 1979 Sensitive in vivo assay of the phenylalanine hydroxylating system with a small intravenous dose of heptadeutero L-phenylalanine using high pressure liquid chromatography and capillary gas chromatography/mass fragmentography. Clin Chim Acta 99:211-230

10. Curtius HC, Zagalak MJ, Baerlocher K, Schaub J, Leimbacher W, Redweik U 1978 In vivo studies of the phenylalanine-4-hydroxylase system in hyperphenylalaninemics and phenylketonurics. Helv Paediatr Acta 32:461-469

11. Treacy EP, Delente JJ, Elkas G, Carter K, Lambert M, Waters PJ, Scriver CR 1997 Analysis of phenylalanine hydroxylase genotypes and hyperphenylalaninemia phenotypes using $\mathrm{L}-\left[1-{ }^{13} \mathrm{C}\right]$ phenylalanine oxidation rates in vivo: a pilot study. Pediatr Res 42:430-435

12. Kure S, Hou DC, Ohura T, Iwamoto H, Suzuki S, Sugiyama N, Sakamoto O, Fujii K, Matsubara Y, Narisawa K 1999 Tetrahydrobiopterin-responsive phenylalanine hydroxylase deficiency. J Pediatr 135:375-378

13. Trefz FK, Aulela-Scholz C, Blau N 2001 Successful treatment of phenylketonuria with tetrahydrobiopterin. Eur J Pediatr 160:315

14. Spaapen LJ, Bakker JA, Velter C, Loots W, Rubio-Gonzalbo ME, Forget PP, Dorland L, De Koning TJ, Poll-The BT, Ploos Van Amstel HK, Bekhof J, Blau N, Duran M 2001 Tetrahydrobiopterin-responsive phenylalanine hydroxylase deficiency in Dutch neonates. J Inherit Metab Dis 24:352-358

15. Nuoffer JM, Thöny B, Romstad A, Blau N 2001 A patient with phenylketonuria successfully treated with tetrahydrobiopterin. J Inherit Metab Dis 24(suppl 1):29

16. Blau N, Trefz FK 2002 Tetrahydrobiopterin-responsive phenylalanine hydroxylase deficiency: possible regulation of gene expression in a patient with the homozygous L48S mutation. Mol Genet Metab 75:186-187

17. Muntau AC, Roschinger W, Habich M, Demmelmair H, Hoffmann B, Sommerhoff CP, Roscher AA 2002 Tetrahydrobiopterin as an alternative treatment for mild phenylketonuria. N Engl J Med 347:2122-2132

18. Ishii T, Takatori K, Iida K, Higuchi T, Ohshima A, Naruse H, Kajiwara M 1998 Optimum conditions for the ${ }^{13} \mathrm{C}$-phenylalanine breath test. Chem Pharm Bull (Tokyo) 46:1330-1332

19. Schoeller DA, Klein PD 1979 A microprocessor controlled mass spectrometer for the fully automated purification and isotopic analysis of breath carbon dioxide. Biomed Mass Spectrom 6:350-355

20. Schofield WN 1985 Predicting basal metabolic rate, new standards and review of previous work. Hum Nutr Clin Nutr 39C(suppl 1):5-41

21. Okano Y, Hase Y, Lee DH, Furuyama J, Shintaku H, Oura T, Isshiki G 1992 Frequency and distribution of phenylketonuric mutations in Orientals. Hum Mutat $1: 216-220$

22. Okano Y, Asada M, Kang Y, Nishi Y, Hase Y, Oura T, Isshiki G 1998 Molecular characterization of phenylketonuria in Japanese patients. Hum Genet 103:613-618

23. Guldberg P, Henriksen KF, Güttler F 1993 Molecular analysis of phenylketonuria in Denmark: $99 \%$ of the mutations detected by denaturing gradient gel electrophoresis. Genomics 7:141-146

24. Nowacki PM, Byck S, Prevost L, Scriver CR 1998 PAH Mutation Analysis Consortium Database: 1997 Prototype for relational locus-specific mutation databases. Nucleic Acids Res 26:220-225

25. Okano Y, Hase Y, Shintaku H, Araki K, Furuyama J-I, Oura T, Isshiki G 1994 Molecular characterization of phenylketonuric mutations in Japanese by analysis of phenylalanine hydroxylase mRNA from lymphoblasts. Hum Mol Genet 3:659

26. Svensson E, Eisensmith RC, Dworniczak B, von Dobeln U, Hagenfeldt L, Horst J, Woo SL 1992 Two missense mutations causing mild hyperphenylalaninemia associated with DNA haplotype 12. Hum Mutat 1:129-137

27. Bernegger C, Blau N 2002 High frequency of tetrahydrobiopterin-responsiveness among hyperphenylalaninemias: a study of 1919 patients observed from 1988 to 2002. Mol Genet Metab 77:304-313

28. Scriver CR 1998 An ongoing debate over phenylalanine hydroxylase deficiency in phenylketonuria. J Clin Invest 101:2613-2614

29. Wang T, Okano Y, Eisensmith RC, Harvey ML, Lo WH, Huang SZ, Zeng YT, Yuan LF, Furuyama JI, Oura T, Sommer SS, Woo SL 1991 Founder effect of a prevalent phenylketonuria mutation in the Oriental population. Proc Natl Acad Sci USA $88: 2146-2150$

30. Wang T, Okano Y, Eisensmith RC, Lo WH, Huang SZ, Zeng YT, Yuan LF, Liu SR, Woo SL 1991 Missense mutations prevalent in Orientals with phenylketonuria: molecular characterization and clinical implications. Genomics 10:449-456 